\title{
Efecto de la suplementación de concentrado fibroso sobre el rendimiento reproductivo de alpacas en altiplano peruano
}

\author{
Effect of fibrous concentrate supplementation on the reproductive performance \\ of alpacas in the Peruvian highlands
}

\author{
Doris Alexandra Rojas ${ }^{1}$, Uri Harold Pérez ${ }^{2}$, Javier Llacsa ${ }^{3}$, Bernardo Roque ${ }^{1}$
}

\section{Resumen}

\begin{abstract}
El objetivo del trabajo fue evaluar el efecto de la suplementación de concentrado fibroso en la dieta sobre el desempeño reproductivo de alpacas en crianza tradicional en la Puna seca los Andes peruanos a $4090 \mathrm{msnm}$, a fin de incrementar la tasa de fertilidad y natalidad, el peso y condición corporal de las madres, y el peso al nacimiento de las crías. Se trabajó con 60 hembras de primer al tercer parto, con similares pesos $(37.8 \pm 1.2 \mathrm{~kg}) \mathrm{y}$ condición corporal $(2.31 \pm 0.42)$, distribuidas en un grupo suplementado y un grupo sin suplementación alimenticia. El concentrado fibroso fue preparado con heno molido (12 mmø) de avena y alfalfa, afrecho de cebada, torta de soya, mezcla vitamínico-mineral ${ }^{(\mathrm{R})}$, sal común y melaza (EM $2.48 \mathrm{kcal} / \mathrm{g}$ y PC $12 \%$ ). El consumo del concentrado se inició 10 días antes de la parición, a razón de $200 \mathrm{~g}$ /día/animal, continuando durante la parición, empadre, gestación, hasta la siguiente parición. El empadre fue controlado, alterno y libre. La tasa de fertilidad se determinó mediante la conducta sexual de las hembras. Los
\end{abstract}

\footnotetext{
${ }^{1}$ Laboratorio de Nutrición Animal, Facultad de Medicina Veterinaria y Zootecnia, Universidad Nacional del Altiplano de Puno, Perú

${ }^{2}$ Laboratorio de Reproducción Animal, Facultad de Medicina Veterinaria y Zootecnia, Universidad Nacional del Altiplano de Puno, Perú

${ }^{3}$ Estación Experimental Agraria Illpa, Dirección de Desarrollo Tecnológico Agrario, Instituto Nacional de Innovación Agraria (INIA), Puno, Perú

${ }^{4}$ E-mail: mvz.llacsa@hotmail.com
}

Recibido: 22 de septiembre de 2020

Aceptado para publicación: 14 de mayo de 2021

Publicado: 24 de agosto de 2021

CLos autores. Este artículo es publicado por la Rev Inv Vet Perú de la Facultad de Medicina Veterinaria, Universidad Nacional Mayor de San Marcos. Este es un artículo de acceso abierto, distribuido bajo los términos de la licencia Creative Commons Atribución 4.0 Internacional (CC BY 4.0) [https:// creativecommons.org/licenses/by/4.0/deed.es] que permite el uso, distribución y reproducción en cualquier medio, siempre que la obra original sea debidamente citada de su fuente original 
resultados indican que la tasa de fertilidad (83.3 vs. 66.7\%), tasa de natalidad (83.3 vs. $50.0 \%$ ), peso de las crías al nacimiento $(5.15 \mathrm{vs} .3 .26 \mathrm{~kg})$, peso final de las madres (44.6 vs. $39.2 \mathrm{~kg}$ ) y condición corporal final de las madres (3.97 vs. 2.77) fueron significativamente mayores con la suplementación $(\mathrm{p}<0.05)$. Se concluye que la suplementación de concentrado fibroso previo a la parición, durante la parición, empadre y gestación tiene un efecto positivo sobre el desempeño reproductivo de las alpacas en crianza tradicional.

Palabras clave: alpacas, concentrado fibroso, nutrición, rendimiento reproductivo, suplementación

\section{AbSTRACT}

The aim of this study was to evaluate the effect of the supplementation of fibrous concentrate in the diet on the reproductive performance of alpacas in traditional breeding in the dry Puna of the Peruvian Andes at $4090 \mathrm{~m}$ of altitude to increase the fertility and birth rate, weight and body condition of the mothers, and weight at birth. In total, 60 females from first to third parity, with similar weights $(37.8 \pm 1.2 \mathrm{~kg})$ and body condition $(2.31 \pm 0.42)$ were distributed into a supplemented group and a group without supplementation. The fibrous concentrate was prepared with ground hay (12 mmø) of oats and alfalfa, barley bran, soybean meal, vitamin and mineral mix, common salt and molasses (ME $2.48 \mathrm{kcal} / \mathrm{g}$ and CP 12\%). The supply of the concentrate started 10 days before calving, at a rate of $200 \mathrm{~g} /$ day/animal, continuing during calving, mating, gestation, until the next calving. The mating was controlled, alternate and free. The fertility rate was determined by the sexual behavior of the females. The results indicate that the fertility rate $(83.3$ vs. $66.7 \%)$, birth rate $(83.3$ vs. $50.0 \%)$, weight at birth $(5.15$ vs. $3.26 \mathrm{~kg})$, final weight of dams (44.6 vs. $39.2 \mathrm{~kg}$ ) and final body condition of dams (3.97 vs. 2.77) were significantly higher with supplementation $(p<0.05)$. It is concluded that the supplementation of fibrous concentrate prior to calving and during breeding and gestation has a positive effect on the reproductive performance of alpacas in traditional breeding.

Key words: alpacas, fibrous concentrate, nutrition, reproductive performance, supplementation

\section{INTRODUCCIÓN}

La ganadería es un componente importante de los medios de subsistencia de los pequeños productores para satisfacer sus necesidades alimenticias y económicas (Assan, 2014; Mahmood et al., 2014), para lo cual la eficiencia reproductiva desempeña una función clave para garantizar la estabilidad y la dinámica de las poblaciones animales (Thornton, 2010; Godber y Wall, 2014); sin embargo, la funcionalidad reproductiva en los camélidos sudamericanos tiene una serie de problemas, principalmente debido a una alta tasa de mortalidad embrionaria. Solo el $50 \%$ de los servicios entre machos y hembras fértiles termina en gestación (Fernández Baca et al., 1970; Olivera et al., 2003; Bravo et al., 2010). La mayor mortalidad embrionaria (58\%) ocurre durante los primeros 30 días de gestación (Bravo y Sumar, 1985; Ratto et al., 2011).

Se ha sugerido una serie de causas de la mortalidad embrionaria, tales como la migración del embrión desde el cuerno derecho al izquierdo (Fernández-Baca et al., 1970); 
Cuadro 1. Requerimientos de energía metabolizable (EM) de alpacas madres en reproducción al pastoreo en pradera nativa de puna seca, en pico de lactación (tres semanas posparto)

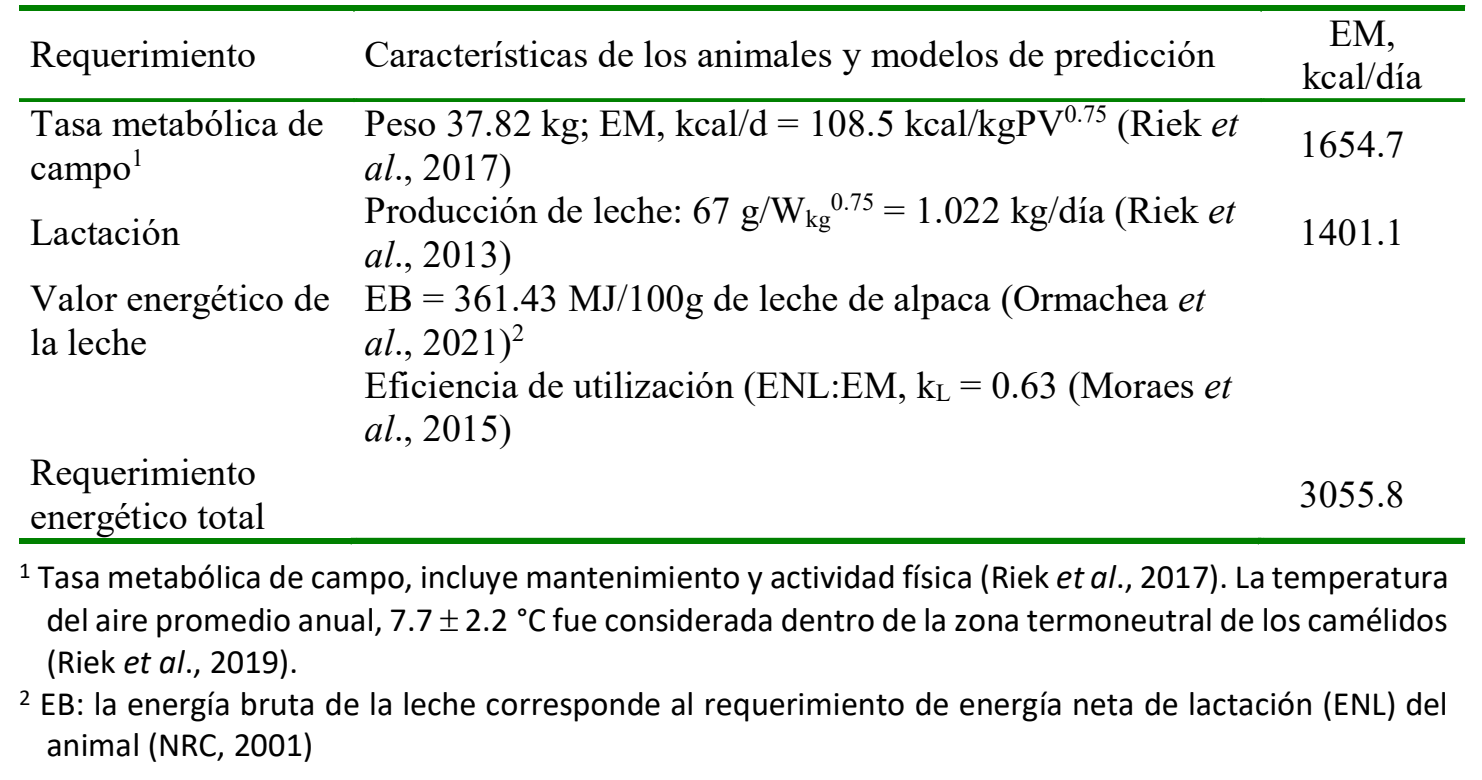

sin embargo, el asunto no ha sido dilucidado. Cervantes et al. (2009) y Llacsa (2012) han mostrado que el problema podría tener un origen nutricional. De esta manera, el presente trabajo tuvo como objetivo determinar el efecto de la suplementación de concentrado fibroso sobre el rendimiento reproductivo de alpacas al pastoreo en praderas naturales en la puna seca de los Andes peruanos, a fin de mejorar las tasas de fertilidad y natalidad, peso y condición corporal de las madres, y el peso de nacimiento de las crías.

\section{Materiales y Métodos}

El trabajo se realizó en el fundo Quiorpatilla, distrito de Ácora, provincia y departamento de Puno, ubicado en puna seca, a una altitud de $4090 \mathrm{~m}$. Se utilizó una muestra de 60 alpacas hembra del primer al tercer parto, procedentes de un sistema de manejo pasto- ril en praderas nativas de Festuca y Calamagrostis (Fedo-Cavi). La pastura presenta típicamente en promedio un valor nutricional de $3.8 \%$ de proteína cruda, $68.6 \%$ de fibra detergente neutro y $2.3 \mathrm{kcal} / \mathrm{g}$ de energía metabolizable en promedio (Mamani et al., 2013) en la época seca; sin embargo, es necesario indicar que los animales fueron selectivos en la dieta, lo cual no fue determinado.

Los animales fueron distribuidos al azar en dos grupos de tratamientos (con y sin suplementación). Los requerimientos energéticos de las alpacas fueron calculados mediante el método factorial, con referencia al peso vivo, la actividad física y la producción de leche (Cuadro 1). El suplemento alimenticio estuvo conformado por un concentrado fibroso preparado con heno de avena y heno de alfalfa, procesados mecánicamente a 12 mmØ de tamaño de partícula, con adición de harina de soya, afrecho de cebada, mezcla mineral comercial, sal común y melaza de 
Cuadro 2. Concentrado fibroso destinado a alpacas madres (base 100\% de materia seca)

\begin{tabular}{lc}
\hline & $\begin{array}{c}\text { Mezcla } \\
(\%)\end{array}$ \\
\hline Heno de avena & 69.00 \\
Heno de alfalfa & 20.00 \\
Torta de soya & 5.00 \\
Afrecho de cebada & 5.00 \\
Mezcla mineral comercial & 0.25 \\
Sal común & 0.25 \\
Melaza de caña & 0.50 \\
\hline Total & 100.0 \\
\hline Valor nutricional de la mezcla \\
$\quad$ EM, kcal/g & 2.18 \\
$\quad$ Proteína cruda, \% mín. & 12.0 \\
Calcio, \% mín. & 0.70 \\
Fósforo, \% mín. & 0.30 \\
$\quad$ Sodio, \% mín. & 0.15 \\
FDN, \% mín. & 48.6 \\
CNF, \% mín & 24.0 \\
\hline
\end{tabular}

EM: energía metabolizable; FDN: fibra detergente neutro; CNF: carbohidratos no fibrosos

caña (Cuadro 2), formulado para contener 12\% de proteína cruda (Van Saun, 2009). .

Los animales pasaron por un periodo de acostumbramiento a la suplementación de siete días. El suplemento fue proporcionado 10 días antes la parición, y se mantuvo durante el empadre, hasta la siguiente parición. El suministro del concentrado fibroso fue a las 06:00 h y en una cantidad diaria de $200 \mathrm{~g}$ por animal, en comederos lineales instalados en el corral de aparto, a una altura superior del alcance de las crías, para consumo colectivo. Los animales pudieron consumir el concentrado durante 1.5 horas y estuvieron en pastoreo durante 8.5 horas. Ambos grupos de alpacas pastorearon juntos y pernoctaron en el mismo corral durante las noches; sin embargo, el grupo con suplementación salía cada mañana al corral de aparto a consumir el concentrado.

El empadre de las alpacas fue controlado, alterno y libre, en una relación de un macho por cada cinco hembras, con un día de trabajo y tres días de descanso por cada macho. La gestación (tasa de fertilidad) se determinó mediante la prueba de la conducta sexual; es decir, rechazo al macho (Raggi et al., 1996; Sumar, 1996; Choque y Ayala, 2017), considerando que esta prueba tiene mucha similitud con los resultados de la ultrasonografía (Alarcón et al., 1990). La tasa de mortalidad embrionaria (o fetal) se determinó mediante la diferencia entre las tasas de fertilidad y natalidad; la condición corporal de las madres fue determinada por estimación de las reservas de grasa corporal a través de la inspección y palpación, con adecuación al puntaje de 1 a 5 (Van Saun, 2013). El peso vivo de las madres fue registrado con balanza electrónica de $100 \pm 0.001$ $\mathrm{kg}$ después de 24 horas del parto. Por otro lado, la tasa de natalidad y el peso de nacimiento de las crías se obtuvo de los registros de nacimientos. Los datos se analizaron mediante la prueba de comparación de medias, con la prueba de $t$ de Student, a un nivel de $\mathrm{a}=0.05$.

\section{Resultados y Discusión}

Los resultados se resumen en el Cuadro 3. Las alpacas con suplementación alimenticia mostraron una mayor tasa de fertilidad $(83.3 \pm 5.8 v s .66 .7 \pm 5.8 \%, \mathrm{p}<0.05)$ y mayor tasa de natalidad que las alpacas sin suplementación ( $83.3 \pm 5.8$ vs. $50.0 \pm 10.0 \%$, $\mathrm{p}<0.05)$. La mortalidad fetal fue nula en alpacas con suplementación, puesto que todas las madres preñadas llegaron con éxito al parto, frente a $16.7 \%$ del grupo sin suplementación. Las pérdidas fetales (abortos) fueron evidentes, principalmente en la 
Cuadro 3. Efecto de la suplementación energética en la dieta sobre la tasa de fertilidad y natalidad, peso inicial, final y condición corporal de las madres $(n=30$ por grupo), peso al nacimiento de las crías en alpacas

\begin{tabular}{lccc}
\hline \multirow{2}{*}{ Variables } & Suplementado. & Sin suplemento & \multirow{2}{*}{$\mathrm{P}_{\text {value }}$} \\
\cline { 2 - 3 } & $\overline{\mathrm{x}} \pm \mathrm{DE}$ & $\overline{\mathrm{x}} \pm \mathrm{DE}$ & \\
\hline Fertilidad, \% & $83.3 \pm 5.8$ & $66.7 \pm 5.8$ & 0.0241 \\
Natalidad, \% & $83.3 \pm 5.8$ & $50.0 \pm 10.0$ & 0.0075 \\
$\begin{array}{l}\text { Peso inicial de las madres, } \mathrm{kg} \\
\text { Peso final de las madres (posparto), }\end{array}$ & $37.8 \pm 1.0$ & $37.9 \pm 1.1$ & 0.9248 \\
$\mathrm{~kg}$ & $44.6 \pm 0.8$ & $39.2 \pm 1.0$ & 0.0017 \\
$\begin{array}{l}\text { Condición corporal inicial de las } \\
\text { madres }\end{array}$ & $2.40 \pm 0.1$ & $2.38 \pm 0.1$ & 0.8149 \\
$\begin{array}{l}\text { Condición corporal final de las } \\
\text { madres }\end{array}$ & $3.97 \pm 0.1$ & $2.77 \pm 0.3$ & 0.0031 \\
\begin{tabular}{l} 
Peso al nacimiento de las crías, $\mathrm{kg}$ \\
\hline
\end{tabular} & $5.15 \pm 0.5$ & $3.26 \pm 0.1$ & 0.0028 \\
\hline
\end{tabular}

época seca (junio y julio), estación caracterizada por las bajas temperaturas y escasez de pastura.

Las alpacas iniciaron con similares pesos y condición corporal, pero finalizaron con valores distintos entre grupos $(44.6 \pm 0.8 v s$. $39.2 \pm 1.0 \mathrm{~kg} ; 3.97 \pm 0.1$ vs. $2.77 \pm 0.3$, respectivamente). En forma similar, las crías de las alpacas con suplementación nacieron con mayores pesos $(\mathrm{p}<0.05)$ que las crías de las alpacas sin suplementación $(5.15 \pm 0.5 \mathrm{vs}$. $3.26 \pm 0.1$, respectivamente). El contraste de los resultados indica que los valores encontrados en el grupo sin suplementación del presente experimento están muy próximos a los valores reportados en la literatura: $29-48 \%$ (Bravo et al., 2010) y 50\% (Fernández-Baca et al., 1970); en cambio, el $833 \%$ de natalidad obtenida en el presente trabajo es mayor que las tasas reportadas de 50\% (Novoa y Leyva, 1996) y de $71.8 \%$ (Huanca et al., 2005).
El peso al nacimiento de las crías de las alpacas con suplementación $(5.15 \pm 0.5 \mathrm{~kg})$ está en el rango de pesos reportados para Perú de 4-10 kg (Bravo, 2014); sin embargo, estos pesos son menores a los observados por Raggi et al. (1997) en Chile. De otro lado, la mayor puntuación de la condición corporal $(3.97 \pm 0.1)$ y el mayor peso final de las alpacas con suplementación $(44.6 \pm 0.8 \mathrm{~kg})$ evidencian una mayor reserva corporal (Vieira et al., 2015), posibilitando una mejor salud, productividad y bienestar animal (Roche et al., 2009).

De las causas de muerte embrionaria sugeridas en la literatura, la nutrición parece constituirse en un factor de importancia. Las alpacas, a diferencia de las demás especies de rumiantes, tienen un alto nivel de glucosa sanguínea (136-183 mg/d) (Siguas et al., 2007) y, por consiguiente, una alta demanda de glucosa, sobre todo en el periparto, cuyo tránsito de gestante a lactante, le impone un 
gran desafío metabólico. En este escenario, la nutrición juega un rol crucial en los distintos eventos que ocurren antes, durante y después de la concepción, tales como la maduración del ovocito, el rendimiento del blastocisto, y la supervivencia prenatal (Ashworth et al., 2009), los cuales dependen de la calidad del ovocito y de la disponibilidad de glucosa (Berlinguer et al., 2012; Evans y Walsh, 2012).

A partir de la teoría desarrollada en rumiantes, se puede indicar que la suplementación incrementa el consumo de materia seca y la provisión de substratos energéticos en el rumen, incrementa la eficiencia de la fermentación ruminal y la captura de nitrógeno para la síntesis de proteína microbial (Agle et al., 2010); incrementa también la producción de ácidos grasos volátiles, con predominio del propiónico, la concentración plasmática de glucosa (Pulido et al., 2007), la secreción de insulina pancreática (Harmon, 1992) y la biosíntesis corporal (Schoenberg et al., 2012); además, mejora el balance energético y disminuye la lipólisis corporal (Pedernera et al., 2008).

\section{Conclusiones}

El suplemento alimenticio a base de concentrado fibroso mostró un efecto positivo sobre el rendimiento reproductivo de las alpacas en crianza tradicional en puna seca, y puede constituirse en una estrategia para el incremento de la fertilidad y natalidad, el peso y condición corporal de las madres, y el peso al nacimiento de las crías.

\section{Agradecimiento}

Los autores agradecen a la señora Isabel Reina Salamanca Paredes, propietaria del Fundo Quiorpatilla-Ácora, por el apoyo logístico con instalaciones y animales para la ejecución de la investigación.

\section{Literatura Citada}

1. Agle M, Hristov AN, Zaman S, Schneider C, Ndegwa PM, Vaddella VK. 2010. Effect of dietary concentrate on rumen fermentation, digestibility, and nitrogen losses in dairy cows. J Dairy Sci 93: 4211-4222. doi: 10.3168/jds.20092977

2. Alarcón V, Sumar J, Riera GS, Foote WC. 1990. Comparison of three methods of pregnancy diagnosis in alpacas and llamas. Theriogenology 34: 1119-1127.

3. Ashworth CJ, Toma LM, Hunter MG 2009. Nutritional effects on oocyte and embryo development in mammals: implications for reproductive efficiency and environmental sustainability. Philos Trans R Soc Lond B Biol Sci 364: 33513361. doi: 10.1098/rstb.2009.0184

4. Assan N. 2014. Micro-livestock farming and food security in Sub Saharan Africa. J Anim Prod Adv 4: 374-387.

5. Berlinguer F, Gonzalez-Bulnes $A$, Contreras-Solis I, Spezzigu A, TorresRovira L, Succu S, Naitana S, et al. 2012. Glucogenic supply increases oocyte developmental competence in sheep. Reprod Fertil Dev 24: 1055-1062. doi: 10.1071/RD11299

6. Bravo PW. 2014. El neonata alpaca, su cuidado y consideraciones inmunológicas. En: XXXVII Reunión Científica Anual de la Asociación Peruana de Producción animal. Abancay, Perú.

7. Bravo PW, Diaz D, Alarcón $V$, Ordoñez C. 2010. Effect of the reproductive state of female alpacas on embryonic mortality rate. Am J Vet Res71: 1096-1099. doi: 10.2460/ ajvr.71.9.1096

8. Bravo W, Sumar J. 1985. Factores que determinan la fertilidad en alpacas. En: V Convención Internacional sobre Camélidos Sudamericanos. Cusco, Perú.

9. Cervantes MP, Orban T, Adams GP. 2009. Ovarian follicular dynamics in south American camelids: effect of plane 
of nutrition and species. Reprod Fert Develop 22: 262-262. doi: 10.1071/ RDv22n1Ab208

10. Choque DS, Ayala C. 2017. Comparación de tres métodos de diagnóstico de preñez en llamas (Lama glama) de la Estación Experimental Choquenaira. Apthapi 3: 1-10.

11. Evans AC, Walsh SW. 2012. The physiology of multifactorial problems limiting the establishment of pregnancy in dairy cattle. Reprod. Reprod Fert Develop 24: 233-237. doi: 10.1071/ RD11912

12. Fernandez-Baca S, Hansel W, Novoa C. 1970. Embryonic mortality in the alpaca. Biol Reprod 3: 243-251. doi: 10.1093/biolreprod/3.2.243

13. Godber OF, Wall R. 2014. Livestock and food security: vulnerability to population growth and climate change. Glob Change Biol 20: 3092-3102. doi: $10.1111 / \mathrm{gcb} .12589$

14. Harmon DL. 1992. Impact of nutrition on pancreatic exocrine and endocrine secretion in ruminants: a review. J Anim Sci 70: 1290-1301. doi: 10.2527/1992.7041290x

15. Huanca W, Ratto $M$, Cordero A, Santiani A, Huanca T, Adams G 2005. Evaluación de un tratamiento de superovulación en la respuesta ovárica y tasa de preñez en llamas. En: XIX Reunión de la Asociación Latinomericana de Producción Animal - ALPA. Tampico, México.

16. Llacsa J. 2012. Efecto de la suplementación energética sobre la eficiencia reproductiva en alpacas Huacaya (Vicugna pacos) con empadre controlado. Tesis de Maestría, Puno, Perú: Unv. Nacional del Altiplano. 67 p.

17. Mahmood HZ, Hussain I, Iftikhar S, Khan M, Nisa FR. 2014. Role of livestock in food security: an ascertainment from Punjab Pakistan. Int $\mathrm{J}$ Acad Res Bus Soc Sci 4: 458-470. doi: 10.6007/IJARBSS/v4-i8/1121
18. Mamani W, Gallo C, Pulido R. 2013. Composición botánica y contenido nutricional de pasturas nativas en periodo seco en el altiplano. En: XXXVIII Congreso Anual Sociedad Chilena de Producción Animal (SOCHIPA). Valdivia, Chile.

19. Moraes L, Kebreab E, Strathe A, Dijkstra J, France J, Casper D, Fadel J. 2015. Multivariate and univariate analysis of energy balance data from lactating dairy cows. J Dairy Sci 98: 4012-4029. doi: 10.3168/jds.2014-8995

20. Novoa C, Leyva V. 1996. Reproducción en alpacas y llamas. Publ Cient IVITA 26: $30 \mathrm{p}$.

21. Olivera LV, Zago DA, Jones CJ, Bevilacqua E. 2003. Developmental changes at the materno-embryonic interface in early pregnancy of the alpaca, Lamos pacos. Anat Embryol 207: $317-$ 331. doi: 10.1007/s00429-003-0346-1

22. Ormachea E, Olarte U, Zanabria V, Melo M, Masias Y. 2021. Composición de la leche de alpaca Huacaya (Vicugna pacos) y de llama (Lama glama). Rev Inv Vet Perú 32: e17800. doi: 10.15381/ rivep.v32i1.17800

23. Pedernera M, García SC, Horagadoga A, Barchia I, Fulkerson WJ. 2008. Energy balance and reproduction on dairy cows fed to achieve low or high milk production on a pasture-based system. J Dairy Sci 91: 3896-3907. doi: 10.3168/jds.2008-1098

24. Pulido RG, Berndt S, Orellana P, Wittwer F. 2007. Effect of source of carbohydrate in concentrate on the performance of high producing dairy cows during spring grazing. Arch Med Vet 39: 19-26.

25. Raggi LA, MacNiven V, Rojas $R$, Castellaro G, Zolezzi M, Latorre E, Parraguez VH, Ferrando G. 1997. Caracterización de la ganancia de peso corporal de alpacas (Lama pacos) des- 
de el nacimiento y hasta los seis meses de edad en cuatro regiones de Chile. Agro Sur 25: 219-226. doi: 10.4206/ agrosur.1997.v25n2-10

26. Raggi L, Ullrich T, Castellaro G, Zolezzi M, Rojas R, Ferrando G, Parraguez H. 1996. Utilización de diferentes métodos de diagnóstico de gestación, en un rebaño experimental de alpacas (Lama pacos) y llamas (Lama glama) en el altiplano de la I región de Chile. Av Cienc Vet 11(1). doi: 10.5354/ 0719-5273.2010.4760

27. Ratto M, Cervantes M, Norambuena C, Silva M, Miragaya M, Huanca $W$. 2011. Effect of location and stage of development of dominant follicle on ovulation and embryo survival rate in alpacas. Anim Reprod Sci 127: 100-105.

28. Riek A, Brinkmann L, Gauly M, Perica J, Ruf T, Arnold W, Gerken M. 2017. Seasonal changes in energy expenditure, body temperature and activity patterns in llamas (Lama glama). Scientific Reports 7(1). doi: 10.1038/ s41598-017-07946-7

29. Riek A, Klinkert A, Gerken M, Hummel J, Moors E, Südekum K. 2013. Short communication: Milk output in llamas (Lama glama) in relation to energy intake and water turnover measured by an isotope dilution technique. J Dairy Sci 96: 1815-1819. doi: 10.3168/jds.2012-6323

30. Riek A, Stölzl A, Bernedo R, Ruf T, Arnold W, Hambly C, Gerken M. 2019. Energy expenditure and body temperature variations in llamas living in the High Andes of Peru. Scientific Reports 9(1). doi: 10.1038/s41598-019-40576-9

31. Roche JR, Friggens NC, Kay JK, Fisher MW, Stafford KJ, Berry DP. 2009. Invited review: body condition score and its association with dairy cow productivity, health, and welfare. J Dairy Sci 92: 5769-5801. doi: 10.3168/jds.20092431

32. Schoenberg KM, Ehrhardt RM, Overton TR. 2012. Effects of plane of nutrition and feed deprivation on insulin responses in dairy cattle during late gestation. J Dairy Sci 95: 670-682. doi: 10.3168/jds.2011-4529

33. Siguas O, Paucar R, Olazabal J, San Martin F, Vélez V. 2007. Valores bioquímicos sanguíneos en alpacas en dos épocas del año en condiciones de Huancavelica: aportes al perfil metabólico de la especie. In: XX Reunión de la Asociación Latinoamericana de Producción Animal. Cusco, Perú.

34. Sumar JB. 1996. Reproduction in llamas and alpacas. Anim Reprod Sci 42: 405-415. doi: 10.1016/0378-4320(96)01538-2

35. Thornton PK. 2010. Livestock production: recent trends, future prospects. Philos T R Soc B 365: 2853-2867. doi: 10.1098/rstb.2010.0134

36. Van Saun RJ. 2009. Nutritional requirements and assessing nutritional status in camelids. Vet Clin Food Anim 25: 265-279. doi: 10.1016/j.cvfa.2009.03.003

37. Van Saun RJ. 2013. Body condition scoring of llamas and alpacas. Penn State Extension, USA. [Internet]. Available in: https://extension.psu.edu/ body-condition-scoring-of-1lamas-andalpacas

38. Vieira A, Brandão $S$, Monteiro A, Ajuda I, Stilwell G. 2015. Development and validation of a visual body condition scoring system for dairy goats with picture-based training. J Dairy Sci 98: 6597-6608. doi: 10.3168/jds.2015-9428 